The Israeli Journal of Aquaculture IJA.73.2021.1429019, 11 pages

CCBY-NC-ND-4.0 • https://doi .org/10.46989/001c.25815

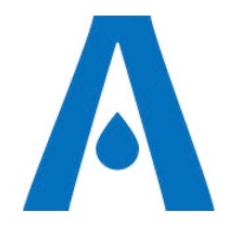

The IJA is a peer-reviewed open-access, electronic journal, freely available without charge to users

Produced by the AquacultureHub non-profit Foundation Sale of IJA papers is strictly forbidden

\title{
Molecular characterization and expression analysis of an antimicrobial peptide, mytimacin-6, in the small abalone, Haliotis diversicolor
}

\author{
Zhilv Xie"\#, Tuo Yao"\#, Jie Lu², Lingtong $\mathrm{Ye}^{2}$, Jiangyong Wang ${ }^{3 *}$ \\ 1 National Demonstration Center for Experimental Fisheries Science Education (Shanghai \\ Ocean University), Shanghai, 201306, China \\ 2 Key Laboratory of South China Sea Fishery Resources Exploitation \& Utilization, \\ Ministry of Agriculture and Rural Affairs, South China Sea Fisheries Research Institute, \\ Chinese Academy of Fishery Sciences, Guangzhou, Guangdong 510300, China \\ ${ }^{3}$ Huizhou University, Huizhou, Guangdong 516001, China
}

Key words: Haliotis diversicolor; Mytimacin 6; Expression analysis; Molecular characterization; Immune response, marine mollusks, abalone,

\begin{abstract}
Macin is a family of antimicrobial peptides (AMPs) and is involved in the immune responses of marine mollusks. In the present study, a novel Mytimacin (denoted as Hd-mtmc 6) was identified from the small abalone Haliotis diversicolor by RNA-seq and RACE techniques. Hd-mtmc 6 contained a coding sequence of $243 \mathrm{bp}$ and encoded 80 amino acids, with a putative peptide mytimacin 6 consisting of 61 amino acid residues. The mature peptide of $\mathrm{Hd}-$ mtmc 6 exhibited typical characteristics of AMPs, including net positive charge $(+4)$, higher hydrophobic residue ratio (37\%), and lower molecular mass. Eight cysteines in the mature peptide formed four disulfide bond bridges (C1-C6, C2$\mathrm{C} 5, \mathrm{C} 3-\mathrm{C} 7$, and C4-C8). Moreover, the presence of the macin domain, a threedimensional structure similar to that of hydramacin-1, and a phylogenetic relationship suggested that Hd-mtmc 6 could be a new member of the invertebrate macin family. In unchallenged abalone, the Hd-mtmc 6 transcript was expressed in all tested tissues and could be detected at different stages of embryonic development. Vibrio harveyi challenge caused a significant Hd-mtmc 6 transcripts upregulation within $2-6 \mathrm{~h}$ in the mantle and hepatopancreas. Our results suggested that $\mathrm{Hd}-\mathrm{mtmc} 6$ may be involved in innate immune responses of small abalone. Further investigations are required to confirm its antibacterial activity at the protein level.
\end{abstract}

* Corresponding author. JiangYong Wang, e-mail: wjy104@163.com

\# These authors contributed equally to this work. 


\section{Introduction}

Marine mollusks inhabit aquatic environments and are constantly exposed to various pathogenic microorganisms (Ammerman et al., 1984). Although mollusks do not secrete immunoglobulins or possess immune memory following the first infection by a pathogen, they have an effective immune defense system (Roch, 1999). Therefore, antimicrobial peptides (AMPs), which constitute the first line of host defense against invading pathogens, are crucial to maintaining homeostasis in mollusks (Liao et al., 2013). AMPs are low molecular weight, cationic, and amphipathic molecules that are evolutionarily conserved (Mihajlovic and Lazaridis, 2010). These properties facilitate AMPs to contact negatively charged bacterial membranes and eventually kill the target bacteria by pore formation or by induction of micellization in a detergent-like manner (Jung et al., 2012). In addition, several AMPs possess other biological activities, including wound healing, promotion of nerve repair, and exerting chemoattraction and cellular immune responses by inducing gene expression (Bals and Wilson, 2003; Jung et al., 2009).

The macin family of AMPs comprises four members, namely theromacin from Theromyzon tessulatum and Hyriopsis cumingii (Tasiemski et al., 2004; Xu et al., 2010), hydramacin from Hydra magnipapillata (Jung et al., 2009), neuromacin from Hirudo medicinalis (Schikorski et al., 2008), and mytimacin from Mytilus galloprovincialis and Achatina fulica (Gerdol et al., 2012; Zhong et al., 2013). Macins are cysteine-rich AMPs characterized by four disulfide bridges formed by eight cysteines. Different macins show various tissue expression patterns. Hydramacin is mainly distributed in the epithelium (Bosch et al., 2009); neuromacin is synthesized by neurons (Schikorski et al., 2008); T. tessulatum theromacin is expressed in large fat cells in contact with coelomic cavities (Tasiemski et al., 2004), while $\mathrm{H}$. cumingii theromacin is distributed in all organs examined, including foot, adductor muscle, hepatopancreas, intestine, mantle, heart, and hemocytes (Xu et al., 2010). Macin transcripts are up-regulated following bacterial challenge (Tasiemski et al., 2004); they are active against a wide range of bacteria and can even inhibit multidrug-resistant bacteria (Jung et al., 2009). The bactericidal action of hydramacin is related to its tertiary structure, which is characterized by a ribbon of residues with a positive charge sandwiched between two hydrophobic areas. Because of this structure, hydramacin promotes bacterial aggregation and change in morphology and eventually kills the bacteria (Jung et al., 2009).

China is a major producer of abalone, which accounts for more than $80 \%$ of the world's total output. The abalone culture industry in China is growing rapidly. Recently, abalone production has exceeded 180,000 tons per year. However, the frequent occurrence of diseases in abalone culture has seriously affected the rapid development of its industry. Presently, the strategies to control abalone diseases mainly rely on the traditional the breeding environments and antibiotic use. The abuse of antibiotics during the occurrence of diseases leads to the development of resistance in pathogenic microorganisms, which increases the difficulty in disease control and affects farmed animals' food safety. In addition, excessive drug residues in aquaculture water will also damage the microbial community structure in the aquatic ecosystem and weaken its ability to degrade organic carbon and nitrogen. AMPs are desirable as potential therapeutic agents for agricultural applications because of their broad-spectrum antibacterial properties and absence of bacterial resistance (Jenssen et al., 2006). Herein, a novel macin family member was identified from the small abalone Haliotis diversicolor (named "Hd-mtmc 6"). The authors determined its molecular structure and the expression pattern in different tissues and at different developmental stages. By using Vibrio harveyi to challenge $H$. diversicolor, we investigated the temporal expression of $\mathrm{Hd}-\mathrm{mtmc} 6$ in the mantle and hepatopancreas. This study helped to understand the molecular mechanism of the immune system of small abalone in response to pathogen infection. It also provided strategies for the sustainable development of abalone culture. 


\section{Materials and Methods}

Ethics statement. $H$. diversicolor is not an endangered or protected species, and there is no requirement for approval to undertake experiments involving this species in China.

Animals, pathogenic bacteria, sample collection, and experimental infection. Small abalone were collected from Daya Bay in Shenzhen (China) and immediately transferred to our laboratory in Guangzhou. The animals were acclimated in $50 \mathrm{~L}$ blue plastic barrels for 2 weeks and maintained in aerated seawater at $26^{\circ} \mathrm{C}$. The animals were fed with Saccharina japonica as their daily diet. Cleaning the experimental barrels took place the following day.

$V$. harveyi, previously from the hepatopancreas of moribund abalone, was used in these experiments. Briefly, $V$. harveyi was inoculated in LB broth at $28^{\circ} \mathrm{C}$ for $18 \mathrm{~h}$. The culture was centrifuged at $8,000 \mathrm{rpm}$ at $4{ }^{\circ} \mathrm{C}$ for $10 \mathrm{~min}$ to obtain the pathogen. The bacterial cells were resuspended in sterile filtered-seawater (SSW) instead of the supernatant fluid, and the bacterial cell concentration was then adjusted to $1.6 \times 10^{6} \mathrm{CFU} / \mathrm{mL}$ for the infection experiment.

The hepatopancreas, mantle, foot, and gill of three healthy abalone individuals were sampled to analyze the tissue expression patterns of Hd-mtmc 6 transcripts. For the expression analysis during larva development, samples from fertilized egg, 32-cell stage, blastula, gastrula, trochophore, veliger, and creeping larva were collected in triplicate. All collected samples were snap-frozen in liquid nitrogen and then stored at $-80^{\circ} \mathrm{C}$ until analysis.

To assess the immune response of $\mathrm{Hd}$-mtmc 6, abalone individuals were infected with $50 \mu \mathrm{L} V$. harveyi through an injection into the foot. The abalone individuals injected with SSW were used as the control group. All experiments were performed in triplicate. The hepatopancreas and mantle of three abalone individuals were randomly collected at 2, 4, $6,12,24,48$, and $72 \mathrm{~h}$ after the challenge. All the collected samples were processed and stored as described above.

RNA isolation and CDNA synthesis. For total RNA extraction and evaluation, an AG RNAex Pro kit (Accurate Biology, Changsha, China) was used to isolate total RNA in accordance with the manufacturer's instructions. A NanoDrop ${ }^{\mathrm{TM}} 2000$ spectrophotometer (Thermo Fisher Scientific, Waltham, MA, USA) was used to detect RNA quantity and purity. In addition, RNA degradation and contamination were monitored by $1 \%$ agarose gel electrophoresis. Only RNA samples with OD260/OD280 ratios of 1.8-2.0 were selected as reverse transcription templates. For the first strand cDNA synthesis, approximately $1 \mu \mathrm{g}$ of total RNA was used as the requirement of the TIANScript ${ }^{T M}$ II Reagent Kit (Tiangen Biotech, Beijing, China).

Gene cloning of Hd-mtmc 6. To obtain the 3'- and 5'-ends of Hd-mtmc 6, four genespecific primers were designed (Table 1) based on the known sequences from the transcriptome database of small abalone. The 5 '-end was obtained by RACE using two nested primers $\mathrm{P} 1$ and $\mathrm{P} 2$. The 3 '-end was obtained using primers $\mathrm{P} 3$ and $\mathrm{P} 4$. For the $\mathrm{Hd}$ mtmc 6 clone, a RACE cDNA Amplification Kit (Clontech, Palo Alto, CA, USA) was used in accordance with the manufacturer's instructions. The PCR products were ligated with the T-A cloning vector (Sangon Biotech, Shanghai, China) and sequenced in both directions. Finally, the 3'- and 5'-ends were combined to obtain the complete cDNA sequence of $\mathrm{Hd}$ mtmc 6.

Bioinformatics analysis. The deduced polypeptide sequences were analyzed with the ExPAsy System (http://www.expasy.org/). The protein domain features of Hd-mtmc 6 were predicted by SMART program (http://smart.embl-heidelberg.de/). The total hydrophobic ratio and net charge were evaluated with APD3 (http://aps.unmc.edu/AP/prediction/prediction_main.php). 3D protein structure prediction of macins was performed using Phyre2 (http://www.sbg.bio.ic.ac.uk/ phyre2/html/page.cgi?id=index).

A multiple sequence alignment of selected sequences was conducted with the Clustal Omega program. The neighbor-joining phylogenetic tree was produced using the MEGA 7.0 program with 1,000 bootstrap replicates. All the macin alignment sequence information 
was downloaded from the GenBank database. The selected amino acid sequences used for the alignment analysis included the following: Hydra vulgaris (Hv hydramacin, AFQ20833), Ruditapes philippinarum hydramacin (Rp hydramacin, AGM14601), R. philippinarum macin (Rp macin, ALJ03313), Scapharca broughtonii macin (Sb macin, AFQ02694), M. galloprovincialis mytimacin-1 (Mg mytimacin-1, CCC15015), M. galloprovincialis mytimacin-2 (Mg mytimacin-2, CCC15016), M. galloprovincialis mytimacin-3 (Mg mytimacin-3, CCC15017), M. galloprovincialis mytimacin-4 (Mg mytimacin-4, CCC15018), M. galloprovincialis mytimacin-5 (Mg mytimacin-5, AHG59340), M. galloprovincialis Mytimacin-6 (Mg Mytimacin-6, AHG59339), H. diversicolor mytimacin-6 (Hd mytimacin-6, QHI06026), Haliotis discus hannai mytimacin-6 (Hdh mytimacin-6, AVW85485), Achatina fulica mytimacin-AF (Af mytimacin-AF, AFR36920), H. medicinalis neuromacin ( $\mathrm{Hm}$ neuromacin BW97519), Aplysia californica theromacin (Ac theromacin ABF21076), $H$. cumingii theromacin (Hc theromacin ADK94899), and Sinanodonta woodiana theromacin (Sw theromacin, AIA62156). The accession numbers are provided in brackets.

Quantitative analysis of Hd-mtmc 6 expression. The expression levels of Hd-mtmc 6 in all samples were determined by relative quantification. All primers were acquired with Primer Premier 5.0, and $\beta$-actin was used as the reference gene to assess the quality of the RNA samples. The primers are shown in Table 1. The SYBR Green Premix Rro Taq HS qPCR Kit (Accurate Biology) was used in qRT-PCR with a Mastercyler ep realplex (Eppendorf, Hamburg, Germany) in accordance with the manufacturer's instructions. PCR was conducted as follows: $95^{\circ} \mathrm{C}$ for $5 \mathrm{~min} ; 40 \mathrm{cycles}$ at $95^{\circ} \mathrm{C}$ for $5 \mathrm{~s}$ and $60{ }^{\circ} \mathrm{C}$ for $30 \mathrm{~s}$. A dissociation curve analysis was performed immediately after 40 PCR cycles. Three biological replicates and three technical replicates were included in these experiments, and the data were obtained using the comparative CT method.

Statistical analysis. One-way analysis of variance (ANOVA) was performed on relative mRNA expression data (presented as mean $\pm S D, n=3$ ) using IBM SPSS 25 . Differences were considered to be significant at $P<0.05$.

Table 1 Primers used in the study of Hd-mtmc 6.

\begin{tabular}{lll}
\hline Primer & Sequences $\left(5^{\prime}-3^{\prime}\right)$ & \multicolumn{1}{c}{ Sequence information } \\
\hline P1 & AGCATCCCTCAGAACACACTTTCCACC & 5' RACE primer out $($ Hd-mtmc 6) \\
P2 & TCAAAGATGCCAGCAGGACAACAGTG & 5' RACE primer in $($ Hd-mtmc 6) \\
P3 & CACTGTTGTCCTGCTGGCATCTTTGA & 3' RACE primer out (Hd-mtmc 6) \\
P4 & GGGTGGAAAGTGTGTCTGAGGGATG & 3' RACE primer in (Hd-mtmc 6) \\
Macin-F & CACTGTCGTCCTGCTGGC & qRT-PCR primer for Hd-mtmc 6 \\
Macin-R & GCTCCTTACACCTGTCACTGC & qRT-PCR primer for Hd-mtmc 6 \\
$\beta-F$ & CCGTGACCTTACAGACTACCT & qRT-PCR primer for $\beta$-actin \\
$\beta-R$ & TACCAGCGGATTCCATAC & qRT-PCR primer for $\beta$-actin \\
\hline
\end{tabular}

\section{Results}

Isolation and analysis of Hd-mtmc 6 cDNA. The complete cDNA sequence of Hd-mtmc 6 (GenBank Accession No: Mk358139) was 572 bp. The 5' untranslated region (UTR) was 98 bp in length. The 3'-UTR contained a consensus polyadenylation signal (AATAAA) and a poly $(A)$ tail, and it was 231 bp in length. The ORF of Hd-mtmc 6 was 243 bp in length and encoded the Hd-mtmc 6 protein of 80 amino acids with a predicted molecular mass of 8.99 $\mathrm{kDa}$. The first 19 amino acids (MFFTVVLLASLMVLPQVEA) were determined as the putative signal peptide. Eight conserved cysteine residues were identified in the macin domain, and they were essential for the formation of a disulfide bridge (Figure 1). The antimicrobial features predicted by the APD3 program affirmed that the mature peptide shared the typical characteristics of AMPs, such as a high percentage of hydrophobic residues $(37 \%)$ and a net positive charge $(+4)$. 
219 AAGTCCTGCAATGACAGGTGTAAGGAGCTTGGCAAATCGGGTGGAAAGTGTGTTCTGAGG $\begin{array}{lllllllllllllllllllll}K & S & C & N & D & R & C & K & E & L & G & K & S & G & G & K & C & V & L & R & 60\end{array}$

279 GATGCTTCAGACTGCTGGATGACAGACAAGGCCTACCAATGTGTTTGCAACAACCGAAAG $\begin{array}{llllllllllllllllllllllll}\mathrm{D} & \mathrm{A} & \mathrm{S} & \mathrm{D} & \mathrm{C} & \mathrm{W} & \mathrm{M} & \mathrm{T} & \mathrm{D} & \mathrm{K} & \mathrm{A} & \mathrm{Y} & \mathcal{C} & \mathrm{C} & \mathrm{V} & \mathrm{C} & \mathrm{N} & \mathrm{N} & \mathrm{R} & \mathrm{K} & 80\end{array}$

339 TAAaacggatccgttttgataattgtatggacgaaatgctcaaattgttttcagttct $-$

399 aaagcaaaacttccgttgtgttttgtccgttaactaacctgacacaagacacctgacatt 459 Ttgtgtctaaactctttccttctataagtatgtttctaaagtatgtgaagttagca 519 aataaaacagatacacgcatttgaaaaaaaaaaaaaaaaaaaaaaaaaaa

Figure 1 Nucleotide and deduced amino acid sequences of Hd-mtmc 6. Initiation and termination codons are shown in red letters. The polyadenylation signal is underlined. The signal peptide is shaded. Cysteines are shown in bold letters.

Multiple sequence alignment and evolutionary tree analysis. A high level of similarity in sequence and structural features was observed across the mollusk macin superfamily members (Figure 2). Sequence similarities between Hd-mtmc 6 and other selected macin family members ranged from $44 \%$ to $80 \%$. The protein sequences of Hd-mtmc 6 showed the highest sequence similarity $(80 \%)$ with the $H$. discus hannai mytimacin 6 precursor (AVW85485). A multiple sequence alignment of the macin superfamily members is shown in Figure 2. The localizations of cysteine in Hd-mtmc 6 were conserved and shared the same disulfide linkages ( $\mathrm{C} 1-\mathrm{C} 6, \mathrm{C} 2-\mathrm{C} 5, \mathrm{C} 3-\mathrm{C} 7$, and $\mathrm{C} 4-\mathrm{C} 8$ ) with other macin family members.

$\mathrm{Hv}$ hydramacin Rp hydramacin $\mathrm{Rp}$ macin Sb macin

Mg mytimacin-1 Mg mytimacin-2 Mg mytimacin-3 Mg mytimacin-4 Mg mytimacin-5 Mg mytimacin-6 Hd mytimacin-6 Hdh mytimacin-6 Af mytimacin-AF $\mathrm{Hm}$ neuromacin Ac theromacin HC theromacin $\mathrm{Sw}$ theromacin

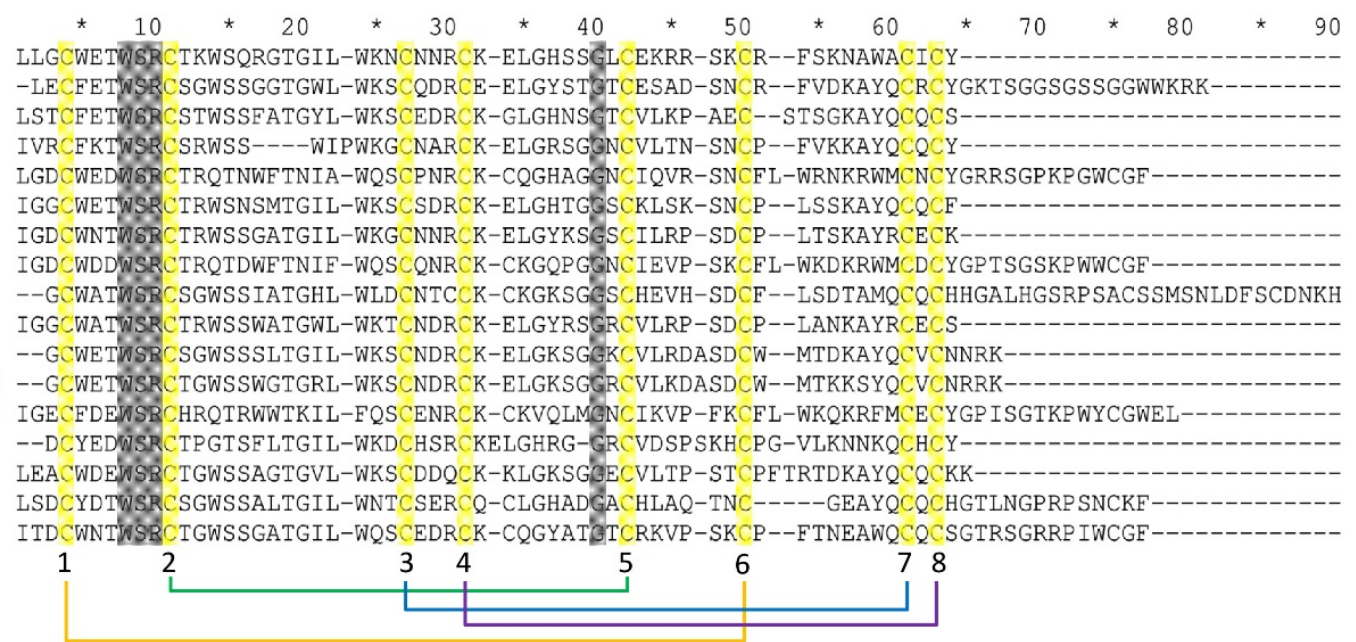

Figure 2 Amino acid sequence alignment of the mature Hd-mtmc 6 sequence with other macin superfamily members. The gray and yellow shaded letters show identical amino acids. Connection lines indicate disulfide linkages.

The tertiary structure of Hd-mtmc 6 modeled by Phyre2 was predicted to be close to that of $H$. vulgaris hydramacin-1 (PDB accession: 2K35) (Figure 3). The two macins share some structural similarities; for example, two $\beta$-sheets, three a-helices, and four identical disulfide bond arrangements were included in their predicted tertiary structure. 
A

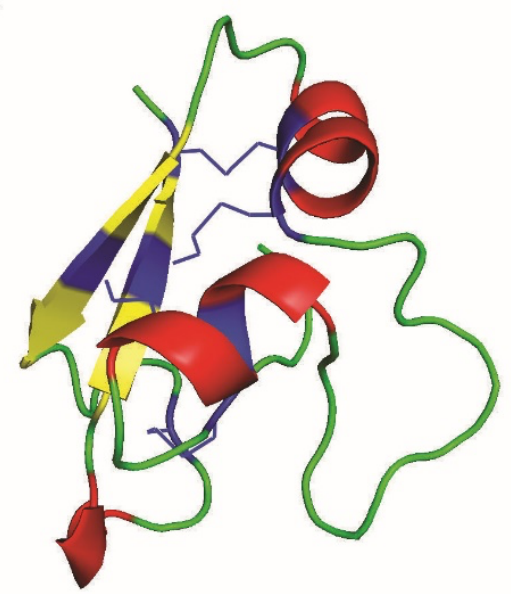

B

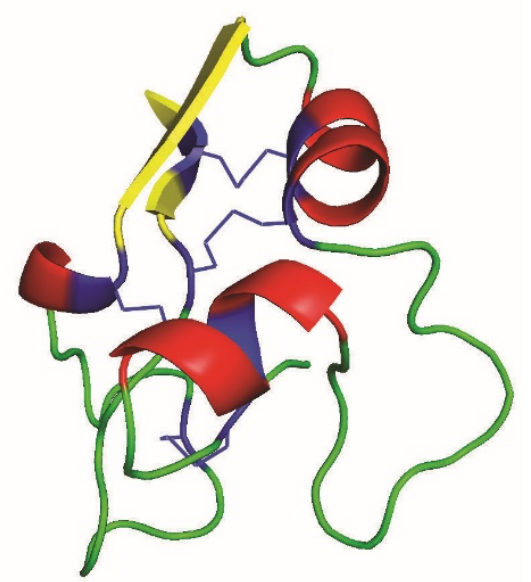

Figure 3 The 3D structures of $H$. vulgaris hydramacin-1 and Hd-mtmc 6. (A) The 3D structure of $H$. vulgaris hydramacin-1 (PDB accession: 2K35). (B) The 3D structure of Hd-mtmc 6 . The eight cysteine residues and four disulfide bridges are shown in blue. The three a-helices are shown in red. The two $\beta$-sheets are shown in yellow.

By using available corresponding invertebrate macin protein sequences, a phylogenetic tree was generated using Mega 7.0 to study the evolutionary relationship. The results showed that four major groups were divided according to these selected protein sequences. In this phylogenetic tree, $\mathrm{Hd}$-mtmc 6 was first clustered with mytimacin 6 from the abalone $H$. discus hannai and then grouped with other macins from mollusks (Figure 4).

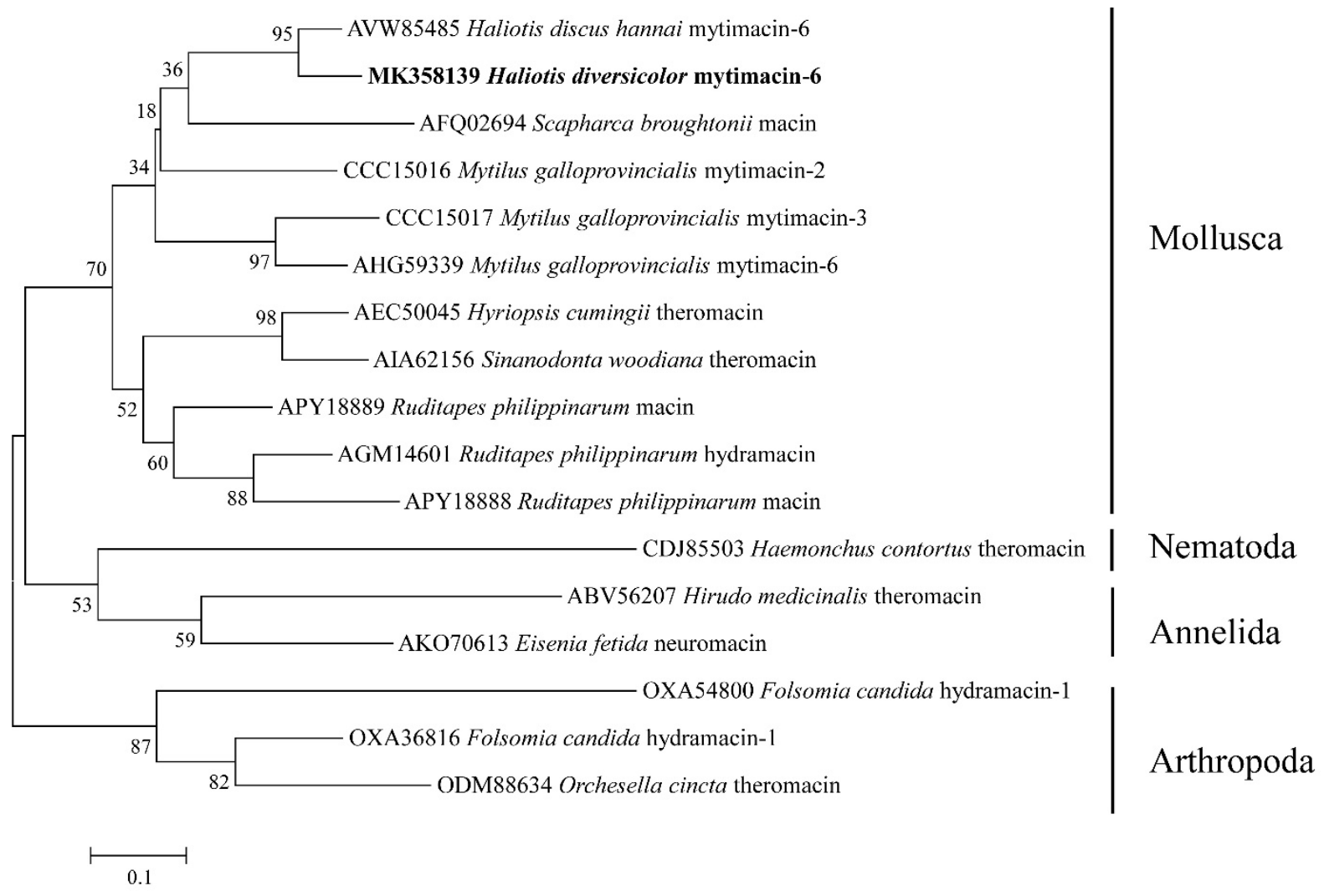

Figure 4 Phylogenetic tree of invertebrate macins. Bootstrap analysis values are shown at the forks $(1,000$ replications). GenBank accession numbers are shown in front of the species. 
Tissue distribution and stage-specific expression patterns of Hd-mtmc 6 mRNA. A qRTPCR analysis was performed to obtain the relative mRNA expression of Hd-mtmc 6 in different tissues, including the mantle, foot, gill, and hepatopancreas. The Hd-mtmc 6 transcripts of $H$. diversicolor were expressed in all the examined tissues. Hd-mtmc 6 transcripts were markedly $(P<0.05)$ higher in the gill, foot, and mantle than in the hepatopancreas, and the highest expression was observed in the mantle (Figure 5A). In addition, the distribution of Hd-mtmc 6 transcripts at different embryonic stages was detected by qRT-PCR. Hd-mtmc 6 transcript was expressed at all stages of embryonic development, including fertilized egg, 32-cell stage, blastula, gastrula, trochophore, veliger, and creeping larva. Moreover, its expression levels fluctuated during embryonic development. In particular, the expression level of Hd-mtmc 6 transcripts gradually increased from the fertilized egg; reached the highest level at the blastocyst and gastrula stage; then significantly declined and increased again at the trochophore and veliger stage, respectively; and finally decreased markedly in the creeping larvae (Figure 5B).
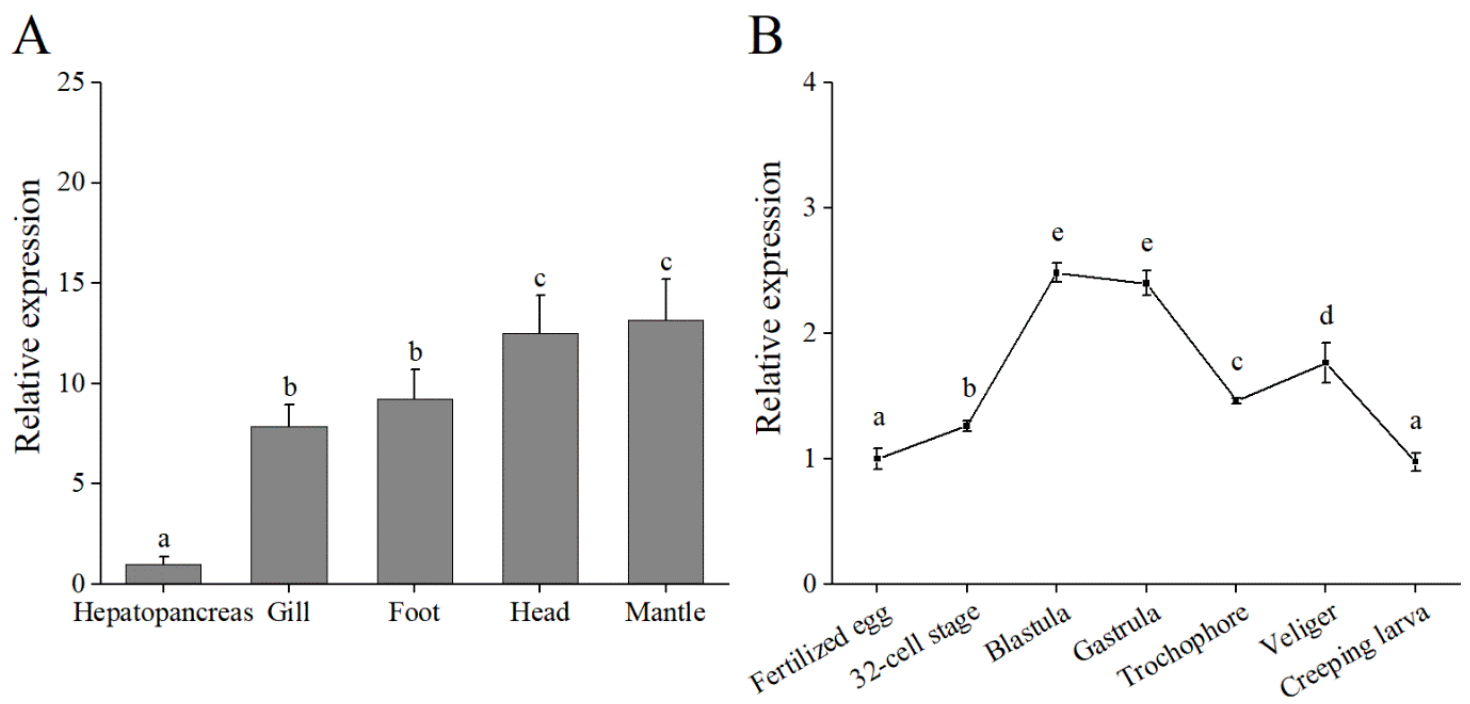

Figure 5 Expression level of Hd-mtmc 6 mRNA in different tissues and developmental stages. (A) Expression level of Hd-mtmc 6 mRNA in different tissues. (B) Expression level of Hd-mtmc 6 mRNA at different developmental stages. Values are expressed as mean \pm SD $(n=3)$. Different letters indicate statistically significant differences $(P<0.05)$.

Expression profile of Hd-mtmc 6 mRNA after $V$. harveyi challenge. The temporal mRNA expression of Hd-mtmc 6 transcript in the mantle and hepatopancreas post $V$. harveyi infection was determined by qRT-PCR analysis, and $\beta$-actin was selected as the reference gene. Hd-mtmc 6 mRNA expression was significantly upregulated in the mantle at 2 and 6 h (Figure 6A). However, Hd-mtmc 6 mRNA expression was significantly increased at 4 and $6 \mathrm{~h}$ in the hepatopancreas (Figure 6B). A significant decrease in mytimacin 6 mRNA was observed at $12 \mathrm{~h}$ in both tissues after bacterial infection. Finally, the mRNA expression of Hd-mtmc 6 was gradually upregulated. 
A

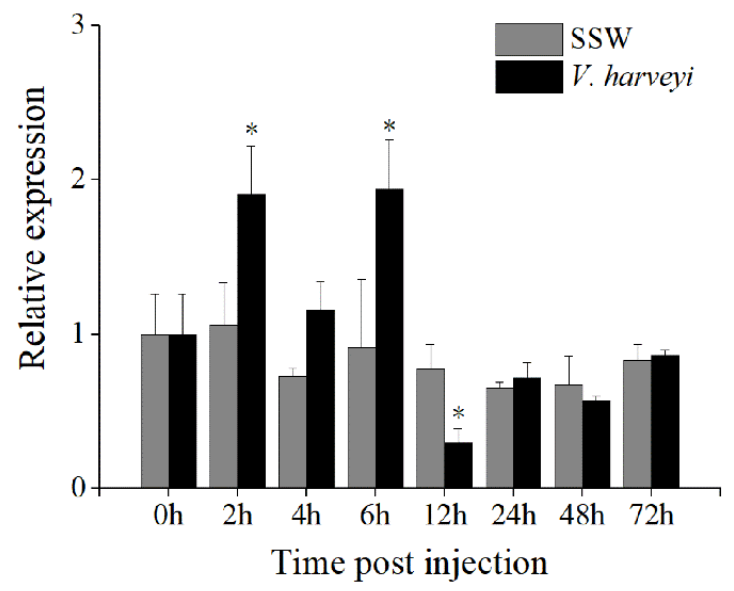

B

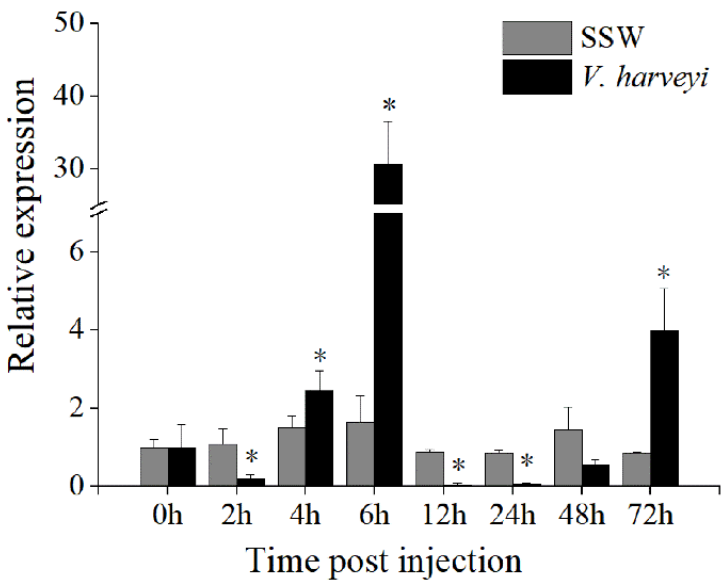

Figure 6 The temporal expression profile of Hd-mtmc 6 mRNA in the mantle (A) and hepatopancreas (B) post $V$. harveyi challenge. Data are expressed as mean $\pm \operatorname{SD}(n=3)$. Asterisks indicate significant differences as compared to the respective control group $(* P<0.05)$.

\section{Discussion}

AMPs have attractive properties because of their biochemical diversity and broad antibacterial activity, and they are less likely to induce resistance. They are considered to be alternatives to synthetic and semi-synthetic antibiotics (Sang and Blecha, 2008). Marine invertebrates have proven to be important resources for obtaining AMPs because of their enormous genetic and biological diversity (Otero-Gonzáiez et al., 2010). In the present study, a novel mytimacin gene in the small abalone $H$. diversicolor was identified by RNAseq and RACE analyses. Hd-mtmc 6 shared several characteristic features of mussel AMPs (Roch et al., 2008), including a high hydrophobic ratio and a net positive charge. The eight cysteine residues involved in the formation of four intramolecular disulfide bridges are conserved in macins (Gerdol et al., 2012). Together with sequence similarity with other macins and structural similarity to hydramacin-1, Hd-mtmc 6 likely acted as an AMP.

In general, the expression pattern of a gene correlates with its functional characteristics. Therefore, a qRT-RCR was performed to detect the expression pattern of Hd-mtmc 6 in different tissues and development stages of investigating its possible function. Hd-mtmc 6 mRNA was constitutively expressed in all the examined tissues in the nonstimulated abalone, including the gill, foot, hepatopancreas, and mantle. These results were similar to the expression pattern of other AMPs found in the ark shell S. broughtonii (Li et al., 2012) and amphioxus Branchiostoma japonicum (Teng et al., 2012), where big defensin was constitutively expressed in most of the tissues examined. AMPs are simultaneously present in different tissues in marine invertebrates, leading to a synergistic immune response and providing a broader spectrum of antimicrobial activity (Sperstad et al., 2011). However, the expression levels of AMPs in different tissues were not consistent. This differential tissue expression pattern indicated their involvement in localized protection.

For example, MGD1 and MGD2 of mussel defensin were solely synthesized and stored in hemocytes (Mitta et al., 1999, 2000). When challenged by bacteria, the peptides were released from the hemocytes. Ark shell big defensin (Sb-BDef1) is abundantly expressed in the hepatopancreas ( $\mathrm{Li}$ et al., 2012). In the present study, the highest expression of Hdmtmc 6 was observed in the mantle, with a higher mRNA level $(13.8$-fold) than that in the hepatopancreas. This was similar to the expression of other AMPs in abalone and oyster defensins (De Zoysa et al., 2010; Gueguen et al., 2006). In fact, the mantle of mollusks is constantly exposed to the aquatic environment and is habitat to multiple bacterial species, some of which are potential pathogens. This indicated that Hd-mtmc 6 played an important role in host defense against environmental microorganisms. The marine environment is rich in bacteria and viruses. Once the gametes of marine shellfish are 
discharged, they are threatened by pathogens. Therefore, it is necessary to develop an effective defense measure. The AMPs are assumed to play an essential role in embryonic development (Liu et al., 2017). In the present study, a constitutive expression of Hd-mtmc 6 was detected at all stages of embryonic development, especially with the highest expression level at the blastula and gastrula stages. This endowed the fertilized eggs of abalone to respond effectively to pathogen invasion at the planktonic stage.

The hepatopancreas is considered an important immune organ because it can synthesize immune factors and initiate immune responses. In addition, the hepatopancreas contains many macrophages and highly specialized cells (Iwanaga and Lee, 2005). Given the important function of the mantle and hepatopancreas, they were selected to investigate the expression level of Hd-mtmc 6 at different time points after bacterial challenge. When challenged with pathogenic $V$. harveyi, Hd-mtmc 6 transcripts were significantly induced in the mantle and hepatopancreas. Similar results have been reported for other AMPs. For example, increased expression of hydramacin and theromacin was detected in the Manila clam (Lee et al., 2014) and the triangle-shell pearl mussel (Xu et al., 2010), respectively. Our preliminary results showed that Hd-mtmc 6 mRNA expression was significantly upregulated in the mantle as early as $2 \mathrm{~h}$ following the challenge with $V$. harveyi, while significantly increased expression was detected at $4 \mathrm{~h}$ in the hepatopancreas. This indicated that the mantle responded more rapidly when infected with a pathogen. Together with the tissue expression pattern of Hd-mtmc 6 transcripts, these findings confirmed the role of the mantle as the first line of defense in immune response (Allam and Pales Espinosa, 2016). Unexpectedly, it was found that the Hd-mtmc 6 mRNA expression was decreased significantly in both the mantle and hepatopancreas at $12 \mathrm{~h}$ post-infection. This finding resembled that observed in Hyriopsis schlegelii and Argopecten irradians (Peng et al., 2012; Zhao et al., 2007). This was thought to be related to the gradual elimination of pathogens. At this time point, the exhaustion of AMPs can be compensated by initiating transcription of other cytokines. The constitutive expression of Hd-mtmc 6 in all the examined tissues and its transcriptional upregulation in the mantle and hepatopancreas after $V$. harveyi challenge suggested that Hd-mtmc 6 may be involved in host defense against pathogens.

Macins have been confirmed to show antibacterial activity at the protein level. In the liquid growth inhibition assay, the purified neuromacin and theromacin were active against gram-positive bacteria. However, no antibacterial activity was found against gramnegative bacteria (Schikorski et al., 2008; Tasiemski et al., 2004). Mytimacin-AF exhibited broad-spectrum antimicrobial activity against Gram-negative and Gram-positive bacteria and fungus (Zhong et al., 2013). Additionally, hydramacin-1 showed higher activity against gram-negative bacteria than against gram-positive bacteria and could kill a large number of multidrug-resistant bacterial strains, including Escherichia coli, Klebsiella pneumoniae, and Klebsiella oxytoca (Bosch et al., 2009; Jung et al., 2009). In the present study, Hdmtmc 6 mRNA could be induced post bacterial infection; however, further studies are required to verify its antimicrobial activity at the protein level.

In summary, we reported Hd-mtmc 6 as a new member of the invertebrate macin family. Hd-mtmc 6 shared higher homology with AMPs of mollusks than with AMPs of other invertebrates. Hd-mtmc 6 was detected in the mantle and in other major tissues and at various developmental stages. Following the challenge with $V$. harveyi, Hd-mtmc 6 transcripts showed an upregulated expression pattern in the mantle and hepatopancreas in a tissue-specific manner. The collective evidence gained from this study suggested that Hd-mtmc 6 played a protective role during the abalone's entire lifespan.

\section{Acknowledgements}

This work was supported by grants from the Science and Technology Planning Project of Guangzhou (202002030488), the China Agriculture Research System of MOF and MARA, the Central Public-interest Scientific Institution Basal Research Fund, CAFS (2020TD42 and 2021SD05), the Science and Technology Planning Project of Jieyang (2019029 and sxm029), the Shellfish and Large Algae Industry Innovation Team Project of Guangdong Province (2020KJ146), the Guangdong Rural Revitalization Strategy Special Funds (Fishery 
Industry Development) (YueCaiNong[2020]4) and the Professorial and Doctoral Scientific Research Foundation of Huizhou University (2020JB065).

\section{References}

Allam, B. and E. Pales Espinosa, 2016. Bivalve immunity and response to infections: Are we looking at the right place? Fish Shellfish Immunol, 53: 4-12. https://doi.org/10.1016/j.fsi.2016.03.037

Ammerman, J., Fuhrman, J., Hagström, Å. and F. Azam, 1984. Bacterioplankton growth in seawater: I. Growth kinetics and cellular characteristics in seawater cultures. Mar Ecol Prog Ser, 18(1-2): 31-39. https://doi.org/10.3354/meps018031

Bals, R. and J.M. Wilson, 2003. Cathelicidins - a family of multifunctional antimicrobial peptides. Cell Mol Life Sci, 60(4): 711-720. https://doi.org/10.1007/s00018-003-2186-9 Bosch, T.C.G., Augustin, R., Anton-Erxleben, F., Fraune, S., Hemmrich, G., Zill, H., Rosenstiel, P., Jacobs, G., Schreiber, S., Leippe, M., Stanisak, M., Grötzinger, J., Jung, S., Podschun, R., Bartels, J., Harder, J. and J.M. Schröder, 2009. Uncovering the evolutionary history of innate immunity: The simple metazoan Hydra uses epithelial cells for host defence. Dev Comp Immunol, 33(4): 559-569. https://doi.org/10.1016/j.dci.2008.10.004

De Zoysa, M., Whang, I., Lee, Y., Lee, S., Lee, J.S. and J. Lee, 2010. Defensin from disk abalone Haliotis discus discus: Molecular cloning, sequence characterization and immune response against bacterial infection. Fish Shellfish Immunol, 28(2): 261-266. https://doi.org/10.1016/j.fsi.2009.11.005

Gerdol, M., De Moro, G., Manfrin, C., Venier, P. and A. Pallavicini, 2012. Big defensins and mytimacins, new AMP families of the Mediterranean mussel Mytilus galloprovincialis. Dev Comp Immunol, 36(2): 390-399. https://doi.org/10.1016/j.dci.2011.08.003

Gueguen, Y., Herpin, A., Aumelas, A., Garnier, J., Fievet, J., Escoubas, J.-M., Bulet, P., Gonzalez, M., Lelong, C., Favrel, P. and E. Bachère, 2006. Characterization of a Defensin from the Oyster Crassostrea gigas. J Biol Chem, 281: 313-323. https://doi.org/10.1074/jbc.M510850200

Iwanaga, S. and B.L. Lee, 2005. Recent Advances in the Innate Immunity of Invertebrate Animals. BMB Rep, 38(2): 128-150. https://doi.org/10.5483/BMBRep.2005.38.2.128

Jenssen, H., Hamill, P. and R.E.W. Hancock, 2006. Peptide Antimicrobial Agents. Clin Microbiol Rev, 19(3): 491-511. https://doi.org/10.1128/CMR.00056-05

Jung, S., Dingley, A.J., Augustin, R., Anton-Erxleben, F., Stanisak, M., Gelhaus, C., Gutsmann, T., Hammer, M.U., Podschun, R., Bonvin, A.M.J.J., Leippe, M., Bosch, T.C.G. and J. Grötzinger, 2009. Hydramacin-1, Structure and Antibacterial Activity of a Protein from the Basal Metazoan Hydra. J Biol Chem, 284(3): 1896-1905. https://doi.org/10.1074/jbc.M804713200

Jung, S., Sönnichsen, F.D., Hung, C.W., Tholey, A., Boidin-Wichlacz, C., Haeusgen, W., Gelhaus, C., Desel, C., Podschun, R., Waetzig, V., Tasiemski, A., Leippe, M. and J. Grötzinger, 2012. Macin Family of Antimicrobial Proteins Combines Antimicrobial and Nerve Repair Activities*. J Biol Chem, 287(17): 14246-14258. https://doi.org/10.1074/jbc.M111.336495

Lee, Y., Revathy, S.K., Lee, S., Whang, I., Oh, C., Kang, D.H., Shin, G.W., Lee, J. and M.D. Zoysa, 2014. First molluscan antimicrobial peptide hydramacin in Manila clam: molecular characterization and expression analysis. J Coast Life Med, 2(6): 447-452.

Li, M., Zhu, L., Zhou, C., Sun, S., Fan, Y. and Z. Zhuang, 2012. Molecular characterization and expression of a novel big defensin (Sb-BDef1) from ark shell, Scapharca broughtonii. Fish Shellfish Immunol, 33(5): 1167-1173. https://doi.org/10.1016/j.fsi.2012.09.008

Liao, Z., Wang, X., Liu, H., Fan, M., Sun, J. and W. Shen, 2013. Molecular characterization of a novel antimicrobial peptide from Mytilus coruscus. Fish Shellfish Immunol, 34(2): 610-616. https://doi.org/10.1016/j.fsi.2012.11.030

Liu, S.H., Wei, D., Yuan, G.R., Jiang, H.B., Dou, W. and J.J. Wang, 2017. Antimicrobial peptide gene cecropin-2 and defensin respond to peptidoglycan infection in the female adult of oriental fruit fly, Bactrocera dorsalis (Hendel). Comp Biochem Physiol Part B 
Biochem Mol Biol, 206: 1-7. https://doi.org/10.1016/j.cbpb.2017.01.004

Mihajlovic, M. and T. Lazaridis, 2010. Antimicrobial peptides bind more strongly to membrane pores. Biochim Biophys Acta - Biomembr, 1798(8): 1494-1502. https://doi.org/10.1016/j.bbamem.2010.02.023

Mitta, G., Hubert, F., Dyrynda, E.A., Boudry, P. and P. Roch, 2000. Mytilin B and MGD2, two antimicrobial peptides of marine mussels: Gene structure and expression analysis. Dev Comp Immunol, 24(4): 381-393. https://doi.org/10.1016/S0145305X(99)00084-1

Mitta, G., Vandenbulcke, F., Hubert, F. and P. Roch, 1999. Mussel defensins are synthesised and processed in granulocytes then released into the plasma after bacterial challenge. J Cell Sci, 112(23): 4233-4242.

Otero - Gonzáiez, A.J., Magalhães, B.S., Garcia - Villarino, M., López - Abarrategui, C., Sousa, D.A., Dias, S.C. and O.L. Franco, 2010. Antimicrobial peptides from marine invertebrates as a new frontier for microbial infection control. FASEB J, 24(5): 1320-1334. https://doi.org/10.1096/fj.09-143388

Peng, K., Wang, J., Sheng, J., Zeng, L. and Y. Hong, 2012. Molecular characterization and immune analysis of a defensin from freshwater pearl mussel, Hyriopsis schlegelii. Aquaculture, 334-337: 45-50. https://doi.org/10.1016/j.aquaculture.2011.12.039

Roch, P., 1999. Defense mechanisms and disease prevention in farmed marine invertebrates. Aquaculture, 172(1-2): 125-145. https://doi.org/10.1016/S00448486(98)00439-6

Roch, P., Yang, Y., Toubiana, M. and A. Aumelas, 2008. NMR structure of mussel mytilin, and antiviral-antibacterial activities of derived synthetic peptides. Dev Comp Immunol, 32(3): 227-238. https://doi.org/10.1016/j.dci.2007.05.006

Sang, Y. and F. Blecha, 2008. Antimicrobial peptides and bacteriocins: alternatives to traditional antibiotics. Anim Heal Res Rev, 9(2): 227-235. https://doi.org/10.1017/S1466252308001497

Schikorski, D., Cuvillier-Hot, V., Leippe, M., Boidin-Wichlacz, C., Slomianny, C., Macagno, E., Salzet, M. and A. Tasiemski, 2008. Microbial Challenge Promotes the Regenerative Process of the Injured Central Nervous System of the Medicinal Leech by Inducing the Synthesis of Antimicrobial Peptides in Neurons and Microglia. J Immunol, 181(2): 1083-1095. https://doi.org/10.4049/jimmunol.181.2.1083

Sperstad, S. V., Haug, T., Blencke, H.M., Styrvold, O.B., Li, C. and, K. Stensvåg, 2011. Antimicrobial peptides from marine invertebrates: Challenges and perspectives in marine antimicrobial peptide discovery. Biotechnol Adv, 29(5): 519-530. https://doi.org/10.1016/j.biotechadv.2011.05.021

Tasiemski, A., Vandenbulcke, F., Mitta, G., Lemoine, J., Lefebvre, C., Sautière, P.E. and M. Salzet, 2004. Molecular Characterization of Two Novel Antibacterial Peptides Inducible upon Bacterial Challenge in an Annelid, the Leech Theromyzon tessulatum. J Biol Chem, 279(30): 30973-30982. https://doi.org/10.1074/jbc.M312156200

Teng, L., Gao, B. and S. Zhang, 2012. The first chordate big defensin: Identification, expression and bioactivity. Fish Shellfish Immunol, 32(4): 572-577. https://doi.org/10.1016/j.fsi.2012.01.007

Xu, Q., Wang, G., Yuan, H., Chai, Y. and Z. Xiao, 2010. cDNA sequence and expression analysis of an antimicrobial peptide, theromacin, in the triangle-shell pearl mussel Hyriopsis cumingii. Comp Biochem Physiol Part B Biochem Mol Biol, 157(1): 119-126. https://doi.org/10.1016/j.cbpb.2010.05.010

Zhao, J., Song, L., Li, C., Ni, D., Wu, L., Zhu, L., Wang, H. and W. Xu, 2007. Molecular cloning, expression of a big defensin gene from bay scallop Argopecten irradians and the antimicrobial activity of its recombinant protein. Mol Immunol, 44(4): 360-368. https://doi.org/10.1016/j.molimm.2006.02.025

Zhong, J., Wang, W., Yang, X., Yan, X. and R. Liu, 2013. A novel cysteine-rich antimicrobial peptide from the mucus of the snail of Achatina fulica. Peptides, 39: 1-5. https://doi.org/10.1016/j.peptides.2012.09.001 